\title{
Nutritional support treatment for perioperative patients in hepatobiliary surgery
}

\author{
Shuifang Jin ${ }^{1}$, Ronglin Jiang ${ }^{1}$, Xi Xing ${ }^{1}$, Weibin $\mathrm{Ma}^{2}$ \\ ${ }^{1}$ Intensive Care Unit, ${ }^{2}$ Department of Pharmacy, First Affiliated Hospital, Zhejiang Chinese Medical University, Hangzhou 310006 , China \\ Correspondence to: Weibin Ma. Department of Pharmacy, First Affiliated Hospital, Zhejiang Chinese Medical University, Hangzhou 310006, China. \\ Email: maweibin96519@126.com. \\ Provenance and Peer Review: This article was commissioned by the editorial office, Hepatobiliary Surgery and Nutrition. The article did not undergo \\ external peer review.
}

Submitted Oct 09, 2019. Accepted for publication Oct 24, 2019.

doi: 10.21037/hbsn.2019.10.27

View this article at: http://dx.doi.org/10.21037/hbsn.2019.10.27

The current principles for medical nutrition support in critically ill patients are early implementation of enteral nutrition, and appropriate supplemental parenteral nutrition when intestinal nutrient is not enough (1). Perioperative patients with hepatobiliary surgery usually have complicated conditions, especially those with complicated cirrhosis, portal hypertension, bile duct obstruction, and diabetes. The nutritional metabolism process and nutritional methods in patients with hepatobiliary surgery are different from other patients and should be treated specially.

The liver is the most important metabolic center organ, and it has a very close relationship with the process of digestion, synthesis and metabolism of nutrients. When liver function is impaired, the above functions are greatly affected. In hepatobiliary surgery, ischemia-reperfusion injury and liver tissue loss are often occurred. These result in a strong stress response, severe hypoproteinemia and metabolic disorders. At this time, we should actively supplement all kinds of nutrients needed for metabolism, but avoid excessive nutrients and lead to metabolic overload. When patients with severe liver damage have reduced ability to metabolize fat, more carbohydrates are needed for energy supply. However, excessive protein will make it prone to azotemia in patients with hepatorenal syndrome.

The basal metabolic rate in some cirrhotic patients is significantly increased, but the efficiency of energy metabolism is significantly reduced (2). This contradiction often shows that energy supply is much lower than energy demand. The body's ability to oxidize glucose is reduced, and protein synthesis is insufficient. But fat oxidation is increased. Patients with cirrhosis often have various degrees of insulin resistance, hyperinsulinemia, and decreased insulin sensitivity, which are less tolerant to glucose (3).

Bile is very important for the digestion and absorption of food, especially for fat. If patients suffer from obstructive jaundice, there are not only abnormalities in intestinal digestion and absorption, but also liver function damage caused by cholestasis. Therefore, they often have various degrees of malnutrition and the lack of essential fatty acids, fat-soluble vitamins, calcium, and phosphorus (4). Moreover, bile acids in bile have a trophic effect on intestinal mucosal epithelial cells, and the mechanical barrier of intestinal mucosal epithelial cells is destroyed in obstructive jaundice (5). The frequent use of antibiotics in biliary obstruction and the micro-ecology in the intestine changes resulted in the destruction of the biological barrier (6). This causes bacteria and toxins in the intestine to easily invade the body and then causes enterogenous infection. Therefore, it is particularly important to actively and steadily implement enteral nutrition to restore the intestinal barrier function.

Nutritional risk screening and nutritional status assessment are particularly important due to the presence of metabolic abnormalities in hepatobiliary diseases. For patients with hepatobiliary surgery, the perioperative period is usually characterized by severe disease, long course of disease, poor nutritional status, and severe stress. These factors result in serious malnutrition risks. A careful malnutrition risk assessment should be performed before surgery, and the nutritional support should be initiated 
early in the presence of malnutrition risk. During the hospitalization, the patient's nutritional status, including muscle strength, blood lipids, protein and albumin, urea nitrogen and creatinine, ammonia, sugar, phosphorus, calcium, lymphocyte count and vitamins should be closely monitored. And the nutrition plan should be adjusted in time according to the test results.

Most patients with hepatobiliary surgery can still implement enteral nutrition (oral or nasal feeding) safely and effectively. On the one hand, enteral nutrition helps to maintain and repair the intestinal barrier; on the other hand, intestinal nutrient absorption through the portal system directly regulates hepatocyte metabolism and effectively promotes hepatocyte regeneration (7). Furthermore, food stimulates the secretion and excretion of bile and improves the state of cholestasis. Therefore, it is necessary to actively apply enteral nutrition in the perioperative period of hepatobiliary surgery.

Enteral nutrition preparations are given before the operation of hepatobiliary surgery, which makes the gastrointestinal tract pre-adapted to enteral nutrition. This is more conducive to recovery of liver function and gastrointestinal function after surgery, and reduces the incidence of postoperative complications. Increased enteral nutrition before surgery can also improve the common hypoproteinemia in patients with hepatobiliary diseases, improve surgical tolerance, and reduce the risk of infection after surgery.

Enteral nutrition begins within $12-24$ h after hepatectomy, which minimizes postoperative complications and promote early recovery (8). The methods should be followed: "Slow first, then fast, step by step" to reduce the occurrence of enteral nutrition intolerance, adjust the speed, concentration and type of preparation of enteral nutrition if necessary.

According to the guidelines (1), critically ill patients should strive to achieve $70 \%$ of their target caloric capacity in 3-7 days after they are tolerant to nutritional support. If not achieved, supplement parenteral nutrition should be given. Hepatobiliary surgery is often in a state of high stress during the perioperative period. Special attention should be paid to the heat/nitrogen ratio and the sugar/fat ratio to facilitate energy metabolism and avoid hyperglycemia. For patients with complicated liver cirrhosis and prone to hepatic encephalopathy, branched-chain amino acids can be administered intravenously or orally $(9,10)$, and blood ammonia levels should be closely monitored. These are beneficial to correct the imbalance of amino acids, reduce the burden on the liver and prevent the occurrence of hepatic encephalopathy.

In summary, the nutritional support has a special nature in the perioperative period of hepatobiliary surgery. It is necessary to actively give various nutrients to meet the metabolism needs. We should pay attention to the changes in metabolic capacity caused by abnormal liver and gallbladder function. Take enteral nutrition as the main factor and be supplemented by parenteral nutrition.

\section{Acknowledgments}

Funding: This study was supported by a grant from the National Natural Science Foundation of China (No. 81774070).

\section{Footnote}

Conflicts of Interest: The authors have no conflicts of interest to declare.

Ethical Statement: The authors are accountable for all aspects of the work in ensuring that questions related to the accuracy or integrity of any part of the work are appropriately investigated and resolved.

Open Access Statement: This is an Open Access article distributed in accordance with the Creative Commons Attribution-NonCommercial-NoDerivs 4.0 International License (CC BY-NC-ND 4.0), which permits the noncommercial replication and distribution of the article with the strict proviso that no changes or edits are made and the original work is properly cited (including links to both the formal publication through the relevant DOI and the license). See: https://creativecommons.org/licenses/by-ncnd/4.0/.

\section{References}

1. Singer P, Blaser AR, Berger MM, et al. ESPEN guideline on clinical nutrition in the intensive care unit. Clin Nutr 2019;38:48-79.

2. Suzuki K, Endo R, Kohgo Y, et al. Guidelines on nutritional management in Japanese patients with liver cirrhosis from the perspective of preventing hepatocellular carcinoma. Hepatol Res 2012;42:621-6.

3. Moctezuma-Velázquez C, García-Juárez I, Soto-Solís R, et al. Nutritional assessment and treatment of patients with 
liver cirrhosis. Nutrition 2013;29:1279-85.

4. Albaugh VL, Banan B, Ajouz H, et al. Bile acids and bariatric surgery. Mol Aspects Med 2017;56:75-89.

5. Pavlidis ET, Pavlidis TE. Pathophysiological consequences of obstructive jaundice and perioperative management. Hepatobiliary Pancreat Dis Int 2018;17:17-21.

6. Faber F, Tran L, Byndloss MX, et al. Host-mediated sugar oxidation promotes post-antibiotic pathogen expansion. Nature 2016;534:697-9.

7. McClave SA, Heyland DK. The physiologic response and associated clinical benefits from provision of early enteral

Cite this article as: Jin S, Jiang R, Xing X, Ma W. Nutritional support treatment for perioperative patients in hepatobiliary surgery. HepatoBiliary Surg Nutr 2020;9(3):342-344. doi: 10.21037/hbsn.2019.10.27 nutrition. Nutr Clin Pract 2009;24:305-15.

8. Rouxel P, Beloeil H. Enhanced recovery after hepatectomy: A systematic review. Anaesth Crit Care Pain Med 2019;38:29-34.

9. Cao Q, Yu CB, Yang SG, et al. Effect of probiotic treatment on cirrhotic patients with minimal hepatic encephalopathy: A meta-analysis. Hepatobiliary Pancreat Dis Int 2018;17:9-16.

10. Gluud LL, Dam G, Les I, et al. Branched-chain amino acids for people with hepatic encephalopathy. Cochrane Database Syst Rev 2017;5:CD001939. 